\title{
Effects of IQP, VEP and Spirulina platensis hydrolysates on the local kidney renin angiotensin system in spontaneously hypertensive rats
}

\author{
JIAHUI ZHENG ${ }^{1,2}$, JINGYUE WANG ${ }^{1}$, HUANGLEI PAN ${ }^{1}$, HONGLI WU ${ }^{1}$, DIFENG REN ${ }^{1}$ and JUN LU ${ }^{2}$ \\ ${ }^{1}$ Beijing Key Laboratory of Forest Food Process and Safety, College of Biological Sciences and Technology, \\ Beijing Forestry University, Beijing 100083; ${ }^{2}$ Beijing Engineering Research Center of Protein and Functional \\ Peptides, China National Research Institute of Food and Fermentation Industries, Beijing 100015, P.R. China
}

Received October 23, 2016; Accepted June 14, 2017

DOI: $10.3892 / \mathrm{mmr} .2017 .7602$

\begin{abstract}
The aim of the present study was to investigate the antihypertensive effects of the bioactive Spirulina platensis peptides Ile-Gln-Pro (IQP), Val-Glu-Pro (VEP), as well as Spirulina platensis hydrolysates ( $\mathrm{SH})$, and assessed whether the synthesis of components of the myocardial and renal local renin angiotensin system (RAS) are regulated differentially in spontaneously hypertensive rats (SHR). The SHR were administrated with IQP, VEP and $\mathrm{SH}$ respectively (10 $\mathrm{mg} / \mathrm{kg} /$ day) for 6 weeks and received continuous monitoring of blood pressure (BP) for two more weeks. During the trial, the rats' kidney tissues were removed from these rats and collected at weeks 3, 6 and 8. The expression of the main components of local kidney RAS was measured at the mRNA levels by reverse transcription-quantitative polymerase chain reaction, and at the protein levels by ELISA or western blotting. Oral administration of IQP, VEP and SH into SHR resulted in marked antihypertensive effects. IQP, VEP and SH decreased rats'
\end{abstract}

Correspondence to: Professor Difeng Ren, Beijing Key Laboratory of Forest Food Process and Safety, College of Biological Sciences and Technology, Beijing Forestry University, 35 East Qinghua Road, Beijing 100083, P.R. China

E-mail: rendifeng@bjfu.edu.cn

Dr Jun Lu, Beijing Engineering Research Center of Protein and Functional Peptides, China National Research Institute of Food and Fermentation Industries, Building 6, Yard 24, Jiuxianqiao Middle Road, Chaoyang, Beijing 100015, P.R. China

E-mail: johnljsmith@163.com

Abbreviations: RAS, renin-angiotensin system; BP, lood pressure; IQP, Ile-Gln-Pro; VEP, Val-Glu-Pro; SH, Spirulina platensis hydrolysates; SHR, spontaneously hypertensive rats; ACE, angiotensin-converting enzyme; Ang II, angiotensin II; AT 1, angiotensin type-1 receptor; RT-qPCR, reverse transcription-quantitative polymerase chain reaction

Key words: Spirulina platensis bioactive peptide, local renin angiotensin system, kidney, myocardium
BP by affecting the expression of local kidney RAS components via downregulating the angiotensin-converting enzyme (ACE), Ang II and angiotensin II (Ang II) and angiotensin type-1 receptor (AT 1), while upregulating ACE2, Ang (1-7), Mas and AT 2. The comparisons of SH effects on local tissue RAS demonstrated that local kidney RAS regulated BP via the ACE-Ang II-AT 1/AT 2 axis and the ACE2-Ang (1-7)-Mas axis primarily at the mRNA level, while the local myocardium RAS mainly at the protein level. This preliminary study suggests that the main components of local RAS presented different expression levels in myocardium and kidney, which is important to the development of bioactive peptides targeting for lowering BP by changing the levels of some components in local RAS in specific tissues.

\section{Introduction}

Hypertension is a global public health issue, which contributes to the burden of heart disease, stroke and kidney failure as well as premature mortality and disability (1). Increasing experimental and clinical evidence supports a key role for the renin-angiotensin system (RAS) in the pathogenesis of hypertension (2). In the RAS, the angiotensin-converting enzyme (ACE2)-Ang (1-7)-Mas axis acts as a counter-regulation system against the ACE-Ang II-AT 1 pathway (3), and changes in the expression and activity of various components of the RAS are implicated in hypertension (4).

In previous years, the focus on the role of the RAS in the pathophysiology of hypertension has changed towards the role of local RAS in specific tissues (5). A previous study reported that in addition to systemic RAS, there is also a local RAS in many tissues, including the kidneys, heart, brain, lungs, liver and blood vessels. Genes of all the components of RAS are expressed in important organs including the heart, brain, kidneys and aorta (6). Research has indicated that tissue RAS can operate independently of circulating RAS (7). Each local RAS has a distinct enzymatic profile resulting in different patterns of angiotensin fragment generation in different tissues (8). Currently, intrarenal RAS is considered to be an important tissue RAS, which controls blood pressure (BP) and is involved in the pathogenesis of hypertension. Appropriate 
activation of the intrarenal RAS ensures kidneys maintain a normal $\mathrm{Na}^{+}$balance at normal renal perfusion pressures to prevent hypertension (9). Regarding intrarenal RAS, the kidney expresses all the major components of the RAS, such as Ang, renin and ACE, suggesting that intrarenal generation of Ang II plays a key role in BP regulation $(10,11)$. Studies indicate that the intrarenal RAS functions separately from systemic Ang II generation (11). In addition, the kidney is one of the most important organs in which Ang (1-7) is generated from the metabolism of Ang II by ACE2, and the proximal tubule exhibits a high level of ACE2 activity. However, the intrarenal effects of the ACE2-Ang (1-7)-Mas receptor pathway are controversial, and further research is required (9).

Alterations in the circadian gene expression of the heart RAS may participate in the development of hypertension (12). Much research was committed to the cardiac RAS $(13,14)$. Fedoseeva et al (15) measured the mRNA expression of kidney and myocardium RAS in hypertensive inbred and normotensive Wistar Albino Glaxo rats respectively, without making analysis and comparison to the mRNA levels between the two tissues local RAS. Further studies should be dedicated to exploring whether the synthesis of components of the myocardial and renal local RAS are differentially regulated after treatment. In a previous study of the authors, they demonstrated the 6-week antihypertensive effects of Ile-Gln-Pro (IQP), Val-Glu-Pro (VEP) and Spirulina platensis hydrolysates (SH) on the local RAS in the myocardium of spontaneously hypertensive rats (SHR). It was identified that ACE, Ang II and AT 1 were downregulated in the myocardium, while AT 2, ACE2, Ang (1-7) and Mas were upregulated in the myocardium (16).

However, how kidney local RAS expression is regulated during the development of antihypertension by IQP, VEP and $\mathrm{SH}$ has not been elucidated. Few studies have explored the differences between the regulation of the kidney and myocardium local RAS to reduce BP although some components of the local RSA of different tissues were determined and analyzed in hypertensive rats $(17,18)$. The current study investigated the effects of intrarenal RAS by examining how IQP, VEP and SH regulated the mRNA levels and the protein concentrations of major components of the RAS [ACE, ACE2, Ang (1-7), Ang II, AT 1, AT 2 and Mas] in the kidney of SHR, and investigated whether $\mathrm{SH}$ had different effects on the BP-reducing mechanisms of the local kidney RAS and local myocardium RAS, using reverse transcription-quantitative polymerase chain reaction, ELISA or western blotting.

\section{Materials and methods}

Reagents. Spirulina platensis powder was purchased from Zaihuishou Bio-engineering (Ererduosi, China, http://nmzhs. com/). IQP and VEP were provided by Beijing SciLight Biotechnology LLC (Beijing, China) with a purity of $>98 \%$. SH was produced by a papain enzymatic method according to the authors' previous studies, and the percentage of IQP and VEP in SH were $\sim 1.81$ and $2.14 \%$, respectively $(16,19,20)$. These three reagents were stored at $-20^{\circ} \mathrm{C}$. Captopril and Nembutal were purchased from YTHX Biotechnology Co., Ltd. (Beijing, China). Coomassie Brilliant Blue Total Protein Quantification kit was purchased from Nanjing Jiancheng Bioengineering Institute (Nanjing, China). ACE ELISA kit (catalog no. ab155452) and ACE2 ELISA kit (catalog no. ab213843) were purchased from Abcam (Cambridge, UK). Ang-II ELISA kit (catalog no. CSB-E07304r) and AT 1 ELISA kit (catalog no. CSB-E13746r) were purchased from Cusabio Biotech., Ltd. (College Park, MD, USA). AT 2 ELISA kit (catalog no. SEA973Ra) and Ang (1-7) ELISA kit (catalog no. CES085Ra) was purchased from Cloud-Clone Corp. (Katy, TX, USA). Unless otherwise stated, all reagents and kits were of analytical grade and were purchased from Tiangen Biotech Co., Ltd. (Beijing, China).

Animal model. A total of 80 male spontaneously hypertensive rats (SHR) purchased from Experimental Animal Center of Weitonglihua (.Beijing,, China, http://www.vitalriver.com/), aged 6 weeks and weighing $235.0 \pm 6.6 \mathrm{~g}$, whose weighted systolic BP was $181 \pm 1 \mathrm{mmHg}$ and weighted diastolic BP was $145 \pm 1 \mathrm{mmHg}$, were used in the present study. Animals were housed in 12-h light/dark cycles under controlled conditions of $25 \pm 2^{\circ} \mathrm{C}$, relative humidity of $60 \pm 5 \%$, and had free access to food and water. These SHR were divided into 5 groups (IQP, VEP, SH, saline and captopril; each $10 \mathrm{mg} / \mathrm{kg} / \mathrm{day}$ ) and received continuous monitoring of BP during six-week treatment period and 2 -week observation period. The administration was conducted by 'passive swallow' gavage instead of usual gavage as to protect the rats' esophagus at 9:00 a.m. every day.

At the end of the first three-week treatment period, rats ( $n=5$, each group) were anesthetized with $3 \%$ pentobarbital (30 mg/kg, intraperitoneal injection). Kidney tissues were removed, cut into small pieces and stored in RNAstore Reagent or in saline for $24 \mathrm{~h}$ at $4^{\circ} \mathrm{C}$, and immediately transferred to an ultra-low-temperature freezer at $-80^{\circ} \mathrm{C}$. Other rats were raised as for the first 3 weeks. At the end of the six-week treatment period, rats ( $n=5$, each group) were euthanized with $3 \%$ pentobarbital (30 mg/kg, intraperitoneal injection). Other rats were raised for another 2 weeks, treated daily with saline, and euthanized as before.

All the cares of the rats were performed in accordance with the Guidelines for the Care and Use of Laboratory Animals, Committee of Beijing Experimental Animal Care and Use. The experiment on the rats was authorized by the Ethics Committee of the Beijing Experimental Animal Association (Beijing, China).

Blood pressure measurement. The systolic BP and diastolic BP were measured twice a week (on Wednesday and Sunday, respectively) at 10:00 a.m. by the tail-cuff method (21) for 8 weeks. Each measurement was repeated five times, and the highest and lowest values were discarded. The mean value was determined for the remaining three values. All measurements were performed by the same person in a quiet environment.

Isolation of RNA from rat kidney. Total RNA from SHR kidneys was isolated using the RNAprep Pure Tissue Kit. RNA purities and concentrations were determined (NanoDrop ND-1000; NanoDrop; Thermo Fisher Scientific, Inc., Wilmington, DE, USA) by the ratio of $A_{260} / A_{280}$ and $A_{260} / A_{230}$.

Single-strand cDNA synthesis. Transcriptor first-strand cDNA was synthesized from $1 \mu \mathrm{g}$ RNA using the First-Strand 
Synthesis kit. An RNA template was placed on ice to defrost, and 5X gDNA Buffer, FQ-RT Primer Mix, 10X Fast RT Buffer and RNase-Free $\mathrm{ddH}_{2} \mathrm{O}$ were placed on ice following thawing at room temperature. Each solution was mixed by vortex oscillation before use and the remaining liquid on the tube wall was collected through brief centrifugation. Then, $2 \mu 15 \mathrm{X}$ gDNA Buffer was added to a thin-walled PCR tube on ice, and 1,000 ng/concentration of RNA (ng/ $\mu \mathrm{l}$ ) total RNA was added. RNA-free water was added to a total volume of $10 \mu \mathrm{l}$. The contents were completely mixed and incubated at $42^{\circ} \mathrm{C}$ for $3 \mathrm{~min}$, and then the reaction tube was placed on ice. The reverse transcription reaction system consisting of $1 \mu \mathrm{l}$ RT Enzyme Mix, $2 \mu$ l FQ-RT Primer Mix, $2 \mu 1$ 10X Fast RT Buffer, $5 \mu \mathrm{l}$ RNase-Free $\mathrm{ddH}_{2} \mathrm{O}$ were added to the thin-walled reaction tube, and the contents were incubated at $42^{\circ} \mathrm{C}$ for $15 \mathrm{~min}$ followed by $95^{\circ} \mathrm{C}$ for another $3 \mathrm{~min}$. The reaction tube was placed on ice and then stored at $-20^{\circ} \mathrm{C}$ for RT-qPCR analysis.

$R T$-qPCR. RT-qPCR was performed for ACE, ACE2, AT 1, AT 2, Mas, and the housekeeping gene GAPDH using the SuperReal PreMix Plus kit. The primers for RT-qPCR analysis were previously reported (16), and were ordered from Tiangen Biotech Co., Ltd. The following experimental operations were performed under dark conditions. All samples were run in triplicate in 96-well plates. PCR reactions were carried out in a $20 \mu 1$ solution consisting of $10 \mu 12 X$ SuperReal PreMix Plus, $0.6 \mu 1$ forward primer $(10 \mu \mathrm{M}), 0.6 \mu 1$ reverse primer $(10 \mu \mathrm{M})$, $4 \mu \mathrm{l}$ cDNA template and $4.8 \mu \mathrm{l}$ RNase-Free $\mathrm{ddH}_{2} \mathrm{O}$. The reaction tube was centrifuged to ensure that all components were at the bottom of the tube. The PCR reaction was initiated with initial denaturation at $95^{\circ} \mathrm{C}$ for $15 \mathrm{~min}$ and then 40 cycles of $10 \mathrm{sec}$ at $95^{\circ} \mathrm{C}$ for denaturation, $20 \mathrm{sec}$ at $60^{\circ} \mathrm{C}$ for annealing, and $32 \mathrm{sec}$ at $72^{\circ} \mathrm{C}$ for extension.

Quantification of a target gene was expressed as the relative expression ratio of the target gene in a sample vs. that of a control (housekeeping gene $G A P D H$ ). The relative quantitative method $\left(2^{-\Delta \Delta \mathrm{Ct}}\right.$ method) was used for the analysis of relative expression ratios of the target genes (22). The value of $2^{-\Delta \Delta \mathrm{Cq}}$ was the relative expression ratio of the target gene.

Western blot analysis. The kidney tissues' total protein was extracted by a Coomassie Brilliant Blue Total Protein Quantification kit following homogeneity $\left(5 \mathrm{~min}, 0^{\circ} \mathrm{C}\right)$ and centrifugation $\left(3,000 \mathrm{x} \mathrm{g}, 10 \mathrm{~min}, 4^{\circ} \mathrm{C}\right)$. The protein samples were separated by $10 \%$ SDS-PAGE, and then transferred to a nitrocellulose membrane. The membrane was blocked with $5 \%$ skimmed milk powder in TBS containing $0.5 \%$ Tween 20 for $2 \mathrm{~h}$ at $37^{\circ} \mathrm{C}$ and then incubated overnight for $2 \mathrm{~h}$ with MAS1L primary antibody (catalog no. ab200685; Abcam) for the Mas receptor at a 1:500 dilution or $G A P D H$ primary antibody (catalog no. sc-47724; Santa Cruz Biotechnology, Inc., Dallas, TX, USA) at a 1:800 dilution for GAPDH. After washing with TBS-T, the blots were then incubated with goat anti-rabbit IgG/HRP secondary antibody (catalog no. ab97040; Abcam) at a 1:3,000 dilution for the Mas receptor or a goat anti-mouse IgG/HRP secondary antibody (catalog no. sc-2005; Santa Cruz Biotechnology, Inc.) at a 1:8,000 dilution for $G A P D H$, then rinsed thoroughly with TBS-T. Proteins were detected by electrochemiluminescence (Bio-Rad Laboratories, Inc., Hercules,
CA, USA). The protein hands were analyzed by Gel Pro 4.0 software (Media Cybernetics, Inc., Rockville, MD, USA).

ELISA. The kidney tissues' total protein content was measured by a Coomassie Brilliant Blue Total Protein Quantification kit following homogeneity $\left(5 \mathrm{~min}, 0^{\circ} \mathrm{C}\right)$ and centrifugation $\left(3,000 \times \mathrm{g}, 10 \mathrm{~min}, 4^{\circ} \mathrm{C}\right)$. Kidney proteins ACE, ACE2, Ang (1-7), Ang II, AT 1 and AT 2 were measured by rat ELISA kits (R\&D Systems, Inc., Minneapolis, MN, USA) according to the manufacturer's instructions. Samples in 96-well microplates were read by a Benchmark plus microplate spectrophotometer (Bio-Rad Laboratories, Inc.) within 20 min after the reaction was stopped. Test samples and standards were measured in duplicate. Microplate manager version 5.2.1 software (Bio-Rad Laboratories, Inc.), which was equipped with the spectrophotometer, automatically calculated sample concentrations according to corresponding standard curves with four-parameter logistics and log-logit function.

Statistical analysis. All values are expressed as the mean \pm standard error of the mean. Statistical comparisons between groups were analyzed with one-factor analysis of variance using SPSS statistical software (version, 17.0; SPSS Inc., Chicago, IL, USA), followed by Tukey's post hoc test. $\mathrm{P}<0.05$ was considered to indicate a statistically significant difference.

\section{Results}

Effects of IQP, VEP and SH on growth parameters and blood pressure changes in SHR. During the eight-week experiment, body weight, consumption of drinking fluid and food of SHR were not significantly different between the groups. As reported previously (16), IQP, VEP and SH had significant anti-hypertensive effects on SHR over an eight-week experimental course.

Effects of IQP, VEP and SH on the ACE-Ang II-AT I/AT 2 axis in local kidney RAS. The qRT-PCR analysis of renal ACE, AT 1 and AT 2 is demonstrated in Fig. 1. In each experimental period (at weeks 3,6 and 8) there was a significant difference in ACE mRNA levels between the every two groups. The $\mathrm{SH}$ group reported the lowest ACE mRNA levels compared with the blank control group (SHR administered saline), except the positive control group (SHR administered captopril). At week 6, when all the experimental groups reached the lowest $\mathrm{BP}$, the ACE mRNA levels of the IQP, VEP and SH group were significantly decreased by 76.80 , 68.60 and $81.70 \%$, respectively, compared with the blank control group (Fig. 1A). AT 1 mRNA expression in renal tissues had a similar trend to ACE. However, at week 6, the AT 1 mRNA level of the VEP group was significantly lower than that of the SH group, and there was no significant difference between the VEP and IQP group. Significant differences of AT $1 \mathrm{mRNA}$ levels were identified at week 6, when compared with week 3 in the same experimental group. At week 6, the AT 1 mRNA levels of the IQP, VEP and SH group were significantly decreased, by 73.00, 74.20 and $70.10 \%$, respectively, compared with the blank control group (Fig. 1B). For AT 2 mRNA levels, there was a significant 

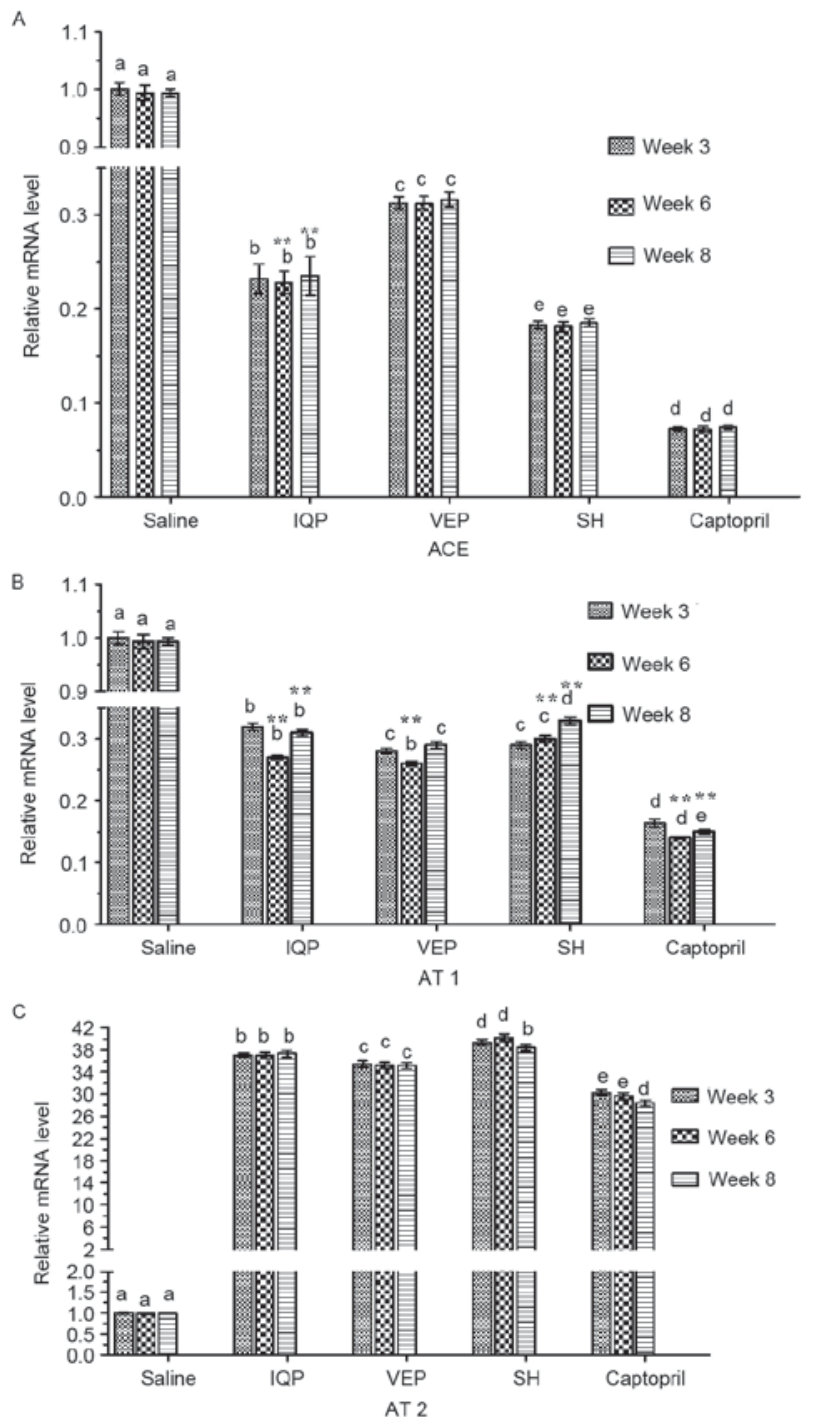

Figure 1. Effects of IQP, VEP and SH on the mRNA levels of the ACE-Ang II-AT 1/AT 2 axis in SHR kidney during different experimental periods (weeks 3, 6, and 8): (A) ACE, (B) AT 1, (C) AT 2. GAPDH was used as a housekeeping gene. Data are represented as the mean \pm standard error of the mean ( $\mathrm{n}=5$ animals per treatment group). In each experimental period, comparisons between different groups were performed by one-way analysis of variance. Values with dissimilar lowercase letters (a-e) were significantly different, $\mathrm{P}<0.05$. Comparisons between different experimental periods of the same treatment group were performed by one-way analysis of variance. ${ }^{* *} \mathrm{P}<0.05$ vs. week 3. IQP, Ile-Gln-Pro; VEP, Val-Glu-Pro; SH, Spirulina platensis hydrolysates; ACE, angiotensin-converting enzyme.

difference between the every two groups at week 6 , when all the experimental groups reached the highest expression of AT 2 mRNA (IQP, VEP and SH group AT 2 mRNA expression was significantly increased by 36.01-, 34.03- and 38.23-fold, respectively, compared with the negative group; Fig. 1C).

ACE, Ang II, AT 1 and AT 2 concentrations in the kidney were measured and quantified by ELISA (Fig. 2). When compared with week 3 , the ACE concentrations of all the experimental groups reached the lowest values at week 6, most notably the IQP and SH group. At week 6, although the ACE concentration of the captopril group was lower than that of $\mathrm{SH}$ group, there was no significant difference between the two groups (Fig. 2A). Ang II presented the same trend. At week 6 , the Ang II concentrations of the IQP, VEP and SH groups were significantly decreased by $32.74,22.10$ and $36.67 \%$, respectively, compared with the blank control group (Fig. 2B). Similar to ACE and Ang II, at week 6, the AT 1 concentrations of the IQP, VEP and SH were significantly decreased by $29.12,21.27$ and $37.37 \%$ respectively, compared with the blank control group. In each experimental period, there was a significant difference in AT 1 concentration between the every two groups (Fig. 2C). AT 2 concentrations increased to a maximum in all the experimental groups at week 6: the AT 2 concentrations in the IQP, VEP and SH groups were significantly increased by $1.08-, 0.74-$ and 1.61 -fold, respectively (Fig. 2D).

Effects of IQP, VEP and SH on ACE2-Ang (1-7)-Mas axis in local kidney RAS. Fig. 3 presents the results of the RT-qPCR analysis of renal ACE2 and Mas mRNA. In each experimental period, there was a significant difference in ACE2 mRNA levels between the every two groups. At week 6, when all the experimental groups reached the highest values, the ACE2 mRNA levels of the IQP, VEP and SH group were significantly increased by 1.31-, 0.91- and 2.01-fold, respectively, compared with the blank control group. Significant differences of ACE2 mRNA levels were identified at week 6, when compared with week 3 in the same experimental group (Fig. 3A). Similar to ACE2, Mas mRNA levels were increased maximally in all the experimental groups at week 6: Mas mRNA levels in IQP, VEP and SH group were significantly increased by 0.76-, 0.51- and 1.68-fold, respectively, compared with the blank control group. At week 8, although the Mas mRNA of captopril group was slightly higher than that of SH group, there was no significant difference between the groups (Fig. 3B).

The ACE2, Ang (1-7) and Mas concentrations in the kidney were measured and quantified by ELISA and western blotting (Fig. 4). In each experimental period, there was a significant difference in ACE2 concentrations between the every two groups. At week 6, the ACE2 concentration levels in the IQP, VEP and SH group were significantly increased by 1.09-, 0.83-, and 1.43-fold, respectively, compared with the blank control group. At week 8, following cessation of administration, the ACE concentrations increased significantly in IQP, VEP and SH groups, respectively, compared to week 3 (Fig. 4A). In each experimental period, the captopril group had the highest level of Ang (1-7) concentration, followed the SH group. Only in the SH group was there a significant increase in Ang (1-7) concentration at week 8 compared with week 3 (Fig. 4B). Mas concentrations in the kidney demonstrated the same trend as Ang (1-7). At week 6, Mas concentrations in the IQP, VEP and SH group were significantly upregulated by 1.68-, 1.41and 2.64-fold, respectively, compared with the blank control group. However, in captopril group, there was a significant decrease in Mas concentration at week 8 compared with week 3 (Fig. 4C).

Effects of SH on the main components in local kidney and myocardium RAS. A comparison of the effects of $\mathrm{SH}$ on ACE, Ang II, AT 1, AT 2, ACE2, Ang (1-7) and Mas at week 6 between local kidney and myocardium local RAS is presented in Fig. 5. For local kidney RAS, the mRNA levels of AT 2, ACE2 and Mas were significantly higher than those in the local myocardium RAS respectively. Of note, the AT 

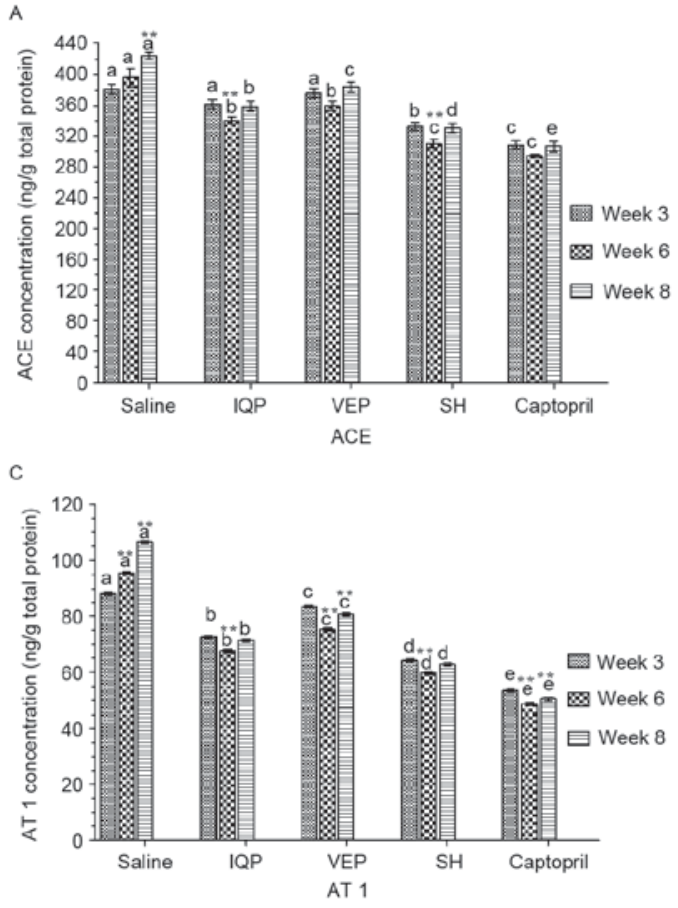

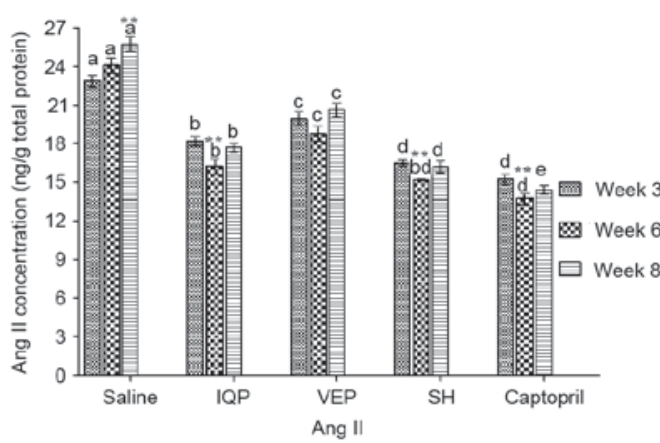

D

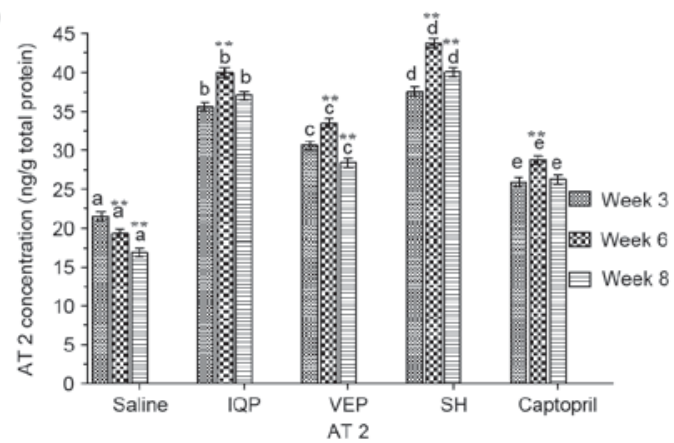

Figure 2. Effects of IQP, VEP and SH on protein concentrations of ACE-Ang II-AT 1/AT 2 axis in the SHR kidney during the different experimental periods (weeks 3, 6, and 8): (A) ACE, (B) Ang II, (C) AT 1, (D) AT 2. Data are represented as mean \pm standard error of the mean (n=5 animals per treatment group). In each experimental period, comparisons between different groups were performed by one-way analysis of variance. Values with dissimilar lowercase letters (a-e) were significantly different, $\mathrm{P}<0.05$. Comparisons between different experimental periods of the same treatment group by one-way analysis of variance. ${ }^{* *} \mathrm{P}<0.05$ vs. week 3. IQP, Ile-Gln-Pro; VEP, Val-Glu-Pro; SH, Spirulina platensis hydrolysates; ACE, angiotensin-converting enzyme.
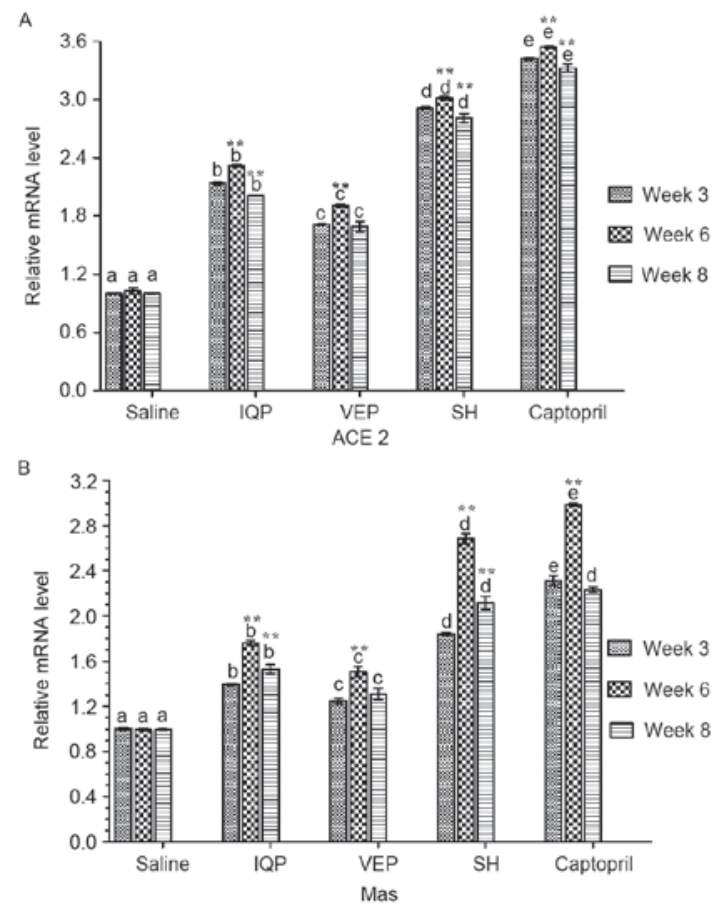

Figure 3. Effects of IQP, VEP and SH on the mRNA levels of ACE2-Ang (1-7)-Mas axis in the SHR kidney during the different during the different experimental periods (weeks 3,6, and 8): (A) ACE2, (B) Mas. GAPDH was the housekeeping gene. Data are represented as mean \pm standard error of the mean ( $\mathrm{n}=5$ animals per treatment group). In each experimental period, comparisons between different groups were performed by one-way analysis of variance. Values with dissimilar lowercase letters (a-e) were significantly different, $\mathrm{P}<0.05$. Comparisons between different experimental periods of the same treatment group by one-way analysis of variance. ${ }^{* *} \mathrm{P}<0.05 \mathrm{vs}$ week 3. IQP, Ile-Gln-Pro; VEP, Val-Glu-Pro; SH, Spirulina platensis hydrolysates; ACE, angiotensin-converting enzyme.
2 mRNA level was 17-fold higher in the local kidney RAS than that in the local myocardium RAS (Fig. 5A). Protein concentrations of ACE and AT 1 in the local kidney RAS were significantly higher than those in the local myocardium RAS, and the concentrations of AT 2, ACE2 and Ang (1-7) were significantly lower than those in the local myocardium RAS (Fig. 5B).

\section{Discussion}

SHR is a well-established model of human essential hypertension. Various studies to determine the antihypertensive effects of food-derived bioactive peptides have used spontaneous hypertensive animals as a model system (23-25). Previous studies by Lu et al $(26,27)$ unraveled the short-term antihypertensive effects of IQP and VEP, as well as the regulation on renin, Ang and other components of the renal ACE-Ang II-AT 1 axis in SHR. Research by Pan et al (16) demonstrated the long-term antihypertensive effects of IQP, VEP and SH in SHR, and explained the effects of these antihypertensive peptides on the expression of local myocardium RAS components. In the current study, the authors investigated the regulative effects of IQP, VEP and SH on the major components of both the ACE-Ang II-AT 1 and ACE2-Ang (1-7)-Mas axes in the local kidney RAS.

In the kidney, all RAS components are present, where both classic and alternate pathways are operational (28). Studies by Lu et al $(26,27)$ demonstrated that one-week IQP and VEP treatment significantly downregulated the mRNA levels of ACE, and AT 1 and upregulated the mRNA expression of AT 2 in the kidney of SHR. The same trend was also reported 

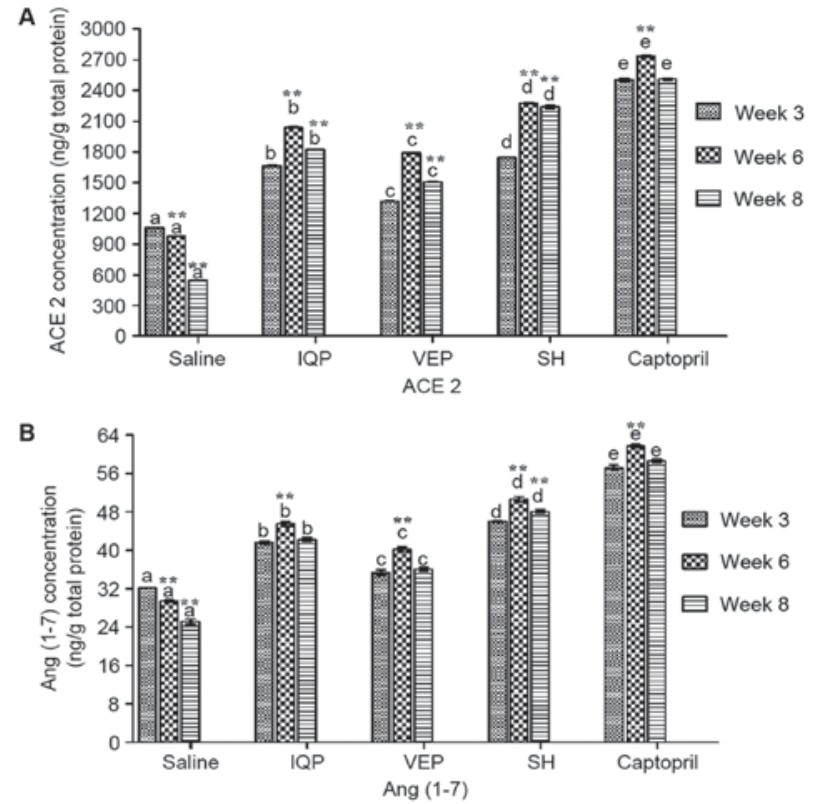

c

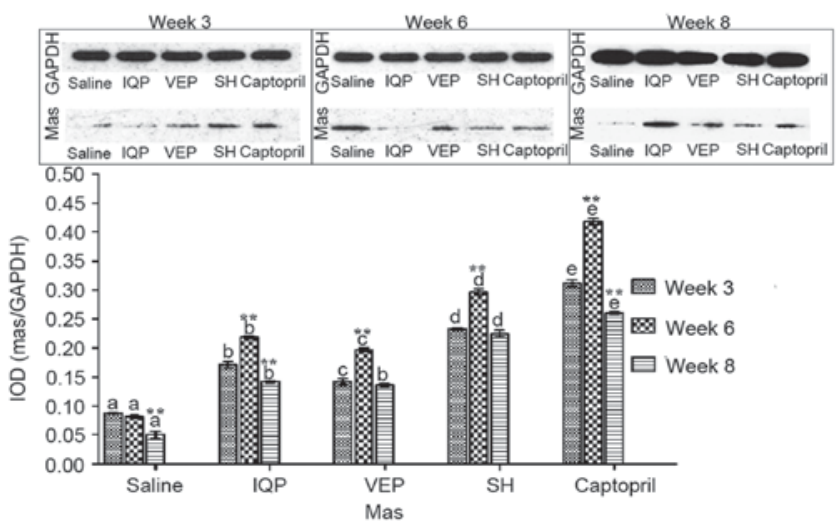

Figure 4. Effects of IQP, VEP and SH on protein concentrations of ACE2-Ang (1-7)-Mas axis in the SHR kidney during the different experimental periods (weeks 3, 6, and 8): (A) ACE2, (B) Ang (1-7), (C) Mas. Data are represented as mean \pm standard error of the mean ( $n=5$ animals per treatment group). In each experimental period, comparisons between different groups were performed by one-way analysis of variance. Values with dissimilar lowercase letters (a-e) were significantly different, $\mathrm{P}<0.05$. Comparisons between different experimental periods of the same treatment group by one-way analysis of variance. ${ }^{* *} \mathrm{P}<0.05$ vs. week 3. IQP, Ile-Gln-Pro; VEP, Val-Glu-Pro; $\mathrm{SH}$, Spirulina platensis hydrolysates; ACE, angiotensin-converting enzyme; Ang, angiotensin.

in this study when comparing ACE, AT 1 and AT 2 mRNA expression at weeks 3 and 6 among the blank control group and the three experimental groups, suggesting there was a close relationship between the antihypertensive effects of IQP, VEP and SH and the downregulation of ACE, and AT 1. Furthermore, the local kidney RAS may be of high relevance for $\mathrm{BP}$ regulation as an amplifier of circulating Ang II actions. Mice generating increased renal Ang II, either by transgenic human RAS or by the local overexpression of rat angiotensinogen, develop high BP (29). Results from the present study indicated that the expression levels of Ang II in the kidney of SHR were significantly reduced following treatment with IQP, VEP or SH, especially at week 6, suggesting that BP reduction was accompanied by lowered Ang II expression levels in the kidney. Therefore, the authors conclude that, for kidney local RAS, suppression of the ACE-Ang II-AT
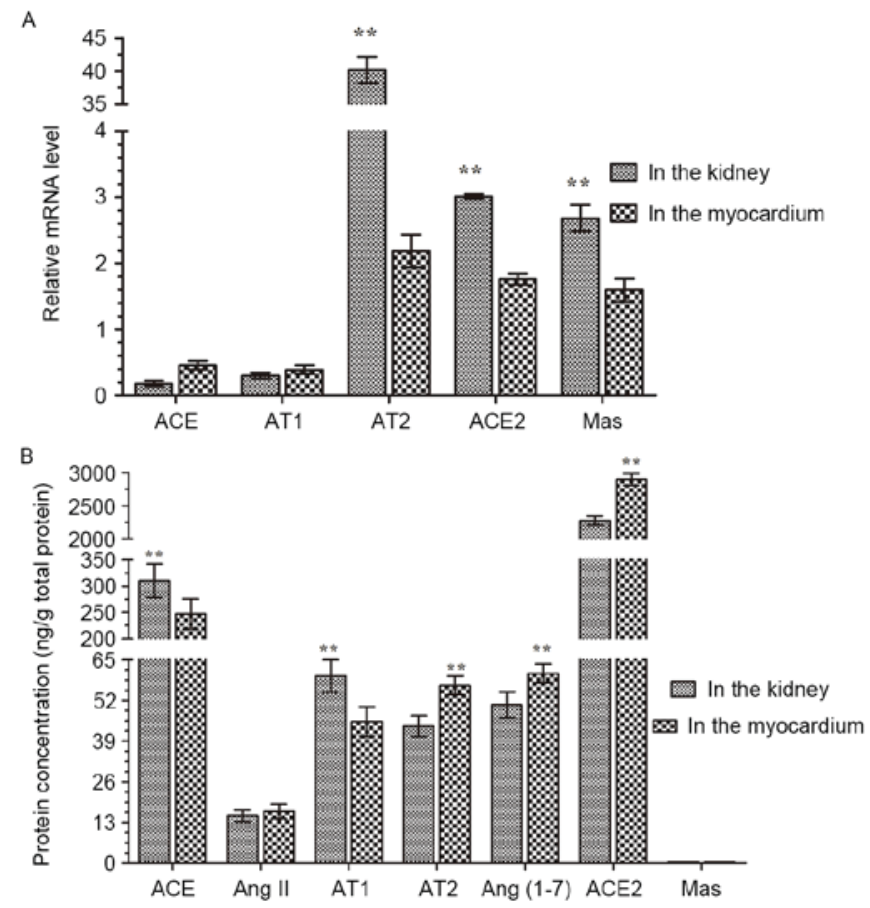

Figure 5. The comparison of the mRNA and protein levels between kidney and myocardium after the six-week treatment of SH. (A) mRNA level, (B) protein concentration. The results are shown as means \pm standard error of the mean $(n=5),{ }^{* *} \mathrm{P}<0.05$ is considered statistically significant by one-way ANOVA between kidney and myocardium after the six-week treatment of $\mathrm{SH}$. ACE, angiotensin-converting enzyme; Ang, angiotensin.

1 axis in the kidney promotes protection against hypertension. Furthermore, most of the evidence suggests that Ang II increases BP by stimulating AT 1, and that AT 2 stimulates a vasodilator signaling cascade that includes bradykinin, nitric oxide and cGMP. AT 2 mediates some of the beneficial actions of AT 1 blockade through this pathway (30). In the present study, at week 6, renal AT 2 mRNA level in the SH group was significantly increased by 38.23 -fold, and the concentration was increased by 1.61-fold compared with the blank control group. Contrary results were observed for AT 1 . These results were similar to those of Yu et al (31). Therefore, the authors propose that, for long-term antihypertensive effects of IQP, VEP and SH, renal AT 2 has an important role in counterbalancing the effects of Ang II mediated by AT 1. In addition, the ACE2-Ang (1-7)-Mas axis is also present in the local kidney RAS. Cervenka et al (32) proposed that the primary mechanism responsible for the BP-lowering effects on chronic hypoxia in Ren-2 transgenic rats was suppression of the hypertensiogenic ACE-Ang II axis in the circulation and kidney tissues, combined with augmentation of the intrarenal vasodilator ACE2-Ang (1-7) axis (32). In the current study, mRNA levels and concentrations of ACE2, Ang (1-7) and Mas in the kidney of the positive control group and experimental groups were significantly upregulated, especially the SH group, in the same experimental period, compared with the blank control group. The results above indicated that IQP, VEP and SH treatments decrease BP, at least in part, by affecting the expression of major local RAS components by downregulating ACE, Ang II and AT 1 while upregulating ACE2, Ang (1-7) and Mas in the kidney of SHR. 
The authors investigated the effect of SH on local tissue RAS (i.e., kidney and myocardium) in SHR following 6 weeks of SH treatment. ACE and AT $1 \mathrm{mRNA}$ levels in the local kidney were significantly lower compared with those in the local myocardium RAS, suggesting that for the local kidney RAS, SH reduces BP by downregulating the ACE-Ang II-AT 1 pathway. The high AT 2 mRNA level in the kidney also suggested that, for the local kidney RAS, AT 2 mRNA level was influenced by SH to reduce the BP. The catabolism of Ang II to produce Ang (1-7) is the main function of ACE2 (33). Although Zhong et al (17) reported a significant upregulation of ACE2 mRNA and protein expression in the heart and kidney, a comparison between the two local RAS was not stated. Other studies reported that ACE2 is highly expressed in the kidney, and its activity is much higher in the kidney cortex than that in heart tissue $(34,35)$. In the current study, the ACE2 mRNA level was significantly higher in the kidney compared with the myocardium, possibly because the pathogenesis and progression of kidney diseases suggest that the renal expression of ACE2 may have a role in controlling local RAS rather than regulating systemic BP (35). Mas mRNA level in the kidney was significantly higher than that in the myocardium, suggesting that kidney local RAS upregulates the ACE2-Ang (1-7)-Mas axis. Therefore, from the mRNA data, the kidney local RAS regulates the two pathways to a greater extent than in the myocardium local RAS. In addition, different components of the RAS are taken up by different tissues, thereby influencing the local synthesis of Ang II (36). However, in the current study, there was no significant difference in Ang II content between the myocardium and kidney local RAS, while the protein levels of Ang (1-7) and ACE2 in myocardium were significantly higher than those in kidney. Accumulating evidence supports the idea that ACE2 is a key negative regulator of the RAS where it metabolizes Ang II into Ang (1-7) (37). Therefore, it was speculated that a decrease of Ang II in the myocardium may be lower than in the kidney, but abundant ACE2 in the myocardium might convert Ang II to Ang (1-7). Moreover, there is good evidence that the ACE2-Ang (1-7)-Mas axis serves an important role in counterbalancing Ang II effects on the heart to maintain normal cardiac function (38). Therefore, from the perspective of the protein levels, in the myocardium local RAS, SH reduced BP primarily by upregulating the ACE2-Ang (1-7)-Mas axis.

Accumulating evidence suggests that local RAS exists in various tissues, which operates independently of their systemic counterparts (39). In the present study, the differences in RAS regulation in the myocardium and kidney were preliminarily compared. Analysis of the expression of various RAS components suggests that the local kidney RAS regulates BP by the ACE-Ang II-AT 1 axis and the ACE2-Ang (1-7)-Mas axis mainly at the mRNA level, while the myocardium local RAS regulates BP mainly at the protein level. However, the specific RAS regulatory mechanism of gene expression in the two organs remains to be determined, and the relationship between the two tissues and circulating RAS is worthy of further investigation.

\section{Acknowledgements}

The present study was supported by grants from the National Natural Science Foundation of China (grant nos. 31571772,
31671963 and 31201339) and the Major State Research Development Program of China (grant no. 2016YFD0400604).

\section{References}

1. WorldHealth Organization(WHO):Aglobalbrief onhypertension. http://apps.who.int/iris/bitstream/10665/79059/1/WHO_DCO WHD_2013.2_eng.pdf. Accessd April 3, 2013.

2. Varagic J, Ahmad S, Nagata $S$ and Ferrario CM: ACE2: Angiotensin II/angiotensin-(1-7) balance in cardiac and renal injury. Curr Hypertens Rep 16: 420, 2014.

3. Iwai $M$ and Horiuchi $M$ : Devil and angel in the renin-angiotensin system: ACE-angiotensin II-AT 1 axis vs. ACE2-angiotensin-(1-7)-Mas receptor axis. Hypertens Res 32: 533-536, 2009

4. Samuel P, Ali Q, Sabuhi R, Wu Y and Hussain T: High Na intake increases renal angiotensin II levels and reduces expression of the ACE2-AT(2)R-MasR axis in obese Zucker rats. Am J Physiol Renal Physiol 303: F412-F419, 2012.

5. Kobori H, Nangaku M, Navar LG and Nishiyama A: The intrarenal renin-angiotensin system: From physiology to the pathobiology of hypertension and kidney disease. Pharmacol Rev 59: 251-287, 2007.

6. Ozkayar N, Dede F, Akyel F, Yildirim T, Ateș I, Turhan T and Altun B: Relationship between blood pressure variability and renal activity of the renin-angiotensin system. J Hum Hypertens 30: 297-302, 2016.

7. Nguyen Dinh Cat A and Touyz RM: A new look at the renin-angiotensin system-focusing on the vascular system. Peptides 32: 2141-2150, 2011.

8. Velez JC: The importance of the intrarenal renin-angiotensin system. Nat Clin Pract Nephrol 5: 89-100, 2009.

9. Carey RM: The intrarenal renin-angiotensin system in hypertension. Adv Chronic Kidney Dis 22: 204-210, 2015.

10. Ferrão FM, Lara LS and Lowe J: Renin-angiotensin system in the kidney: What is new? World J Nephrol 3: 64-76, 2014.

11. Giani JF, Shah KH, Khan Z, Bernstein EA, Shen XZ, McDonough AA, Gonzalez-Villalobos RA and Bernstein KE: The intrarenal generation of angiotensin II is required for experimental hypertension. Curr Opin Pharmacol 21: 73-81, 2015.

12. Naito $Y$, Tsujino T, Fujioka $Y$, Ohyanagi $M$ and Iwasaki T: Augmented diurnal variations of the cardiac renin-angiotensin system in hypertensive rats. Hypertension 40: 827-833, 2002.

13. Re RN: The clinical implication of tissue renin angiotensin systems. Curr Opin Cardiol 16: 317-327, 2001.

14. Paul M, Poyan Mehr A and Kreutz R: Physiology of local renin-angiotensin systems. Physiol Rev 86: 747-803, 2006.

15. Fedoseeva LA, Riazanova MA, Antonov EV, Dymshits GM and Markel' AL: Renin-angiotensin system gene expression in the kidney and in the heart in hypertensive ISIAH rats. Biomed Khim 57: 410-419, 2011 (In Russian).

16. Pan H, She X, Wu H, Ma J, Ren D and Lu J: Long-term regulation of the local renin-angiotensin system in the myocardium of spontaneously hypertensive rats by feeding bioactive peptides derived from spirulina platensis. J Agric Food Chem 63: 7765-7774, 2015.

17. Zhong JC, Huang DY, Yang YM, Li YF, Liu GF, Song XH and Du K: Upregulation of angiotensin-converting enzyme 2 by all-trans retinoic acid in spontaneously hypertensive rats. Hypertension 44: 907-912, 2004.

18. Malikova E, Galkova K, Vavrinec P, Vavrincova-Yaghi D, Kmecova Z, Krenek P and Klimas J: Local and systemic renin-angiotensin system participates in cardiopulmonary-renal interactions in monocrotaline-induced pulmonary hypertension in the rat. Mol Cell Biochem 418: 147-157, 2016.

19. Lu J, Ren DF, Xue YL, Sawano Y, Miyakawa T and Tanokura M: Isolation of an antihypertensive peptide from alcalase digest of spirulina platensis. J Agric Food Chem 58: 7166-7171, 2010.

20. Lu J, Ren DF, Wang JZ and Tanokura M: Purification and characterization of an angiotensin I-converting enzyme inhibitory peptide derived from Spirulina platensis. Prog Biochem Biophys 37: 568-574, 2010.

21. Buñag RD: Validation in awake rats of a tail-cuff method for measuring systolic pressure. J Appl Physiol 34: 279-282, 1973.

22. Livak KJ and Schmittgen TD: Analysis of relative gene expression data using real-time quantitative PCR and the 2(-Delta Delta C(T)) method. Methods 25: 402-408, 2001.

23. Siragy HM: AT(1) and AT(2) receptors in the kidney: Role in disease and treatment. Am J Kidney Dis 36 (3 Suppl 1): S4-S9, 2000. 
24. Marczak ED, Usui H, Fujita H, Yang Y, Yokoo M, Lipkowski AW and Yoshikawa M: New antihypertensive peptides isolated from rapeseed. Peptides 24: 791-798, 2003.

25. Igarashi K, Yoshioka K, Mizutani K, Miyakoshi M, Murakami T and Akizawa T: Blood pressure-depressing activity of a peptide derived from silkworm fibroin in spontaneously hypertensive rats. Biosci Biotechnol Biochem 70: 517-520, 2006.

26. Lu J, Sawano Y, Miyakawa T, Xue YL, Cai MY, Egashira Y, Ren DF and Tanokura M: One-week antihypertensive effect of Ile-Gln-Pro in spontaneously hypertensive rats. J Agric Food Chem 59: 559-563, 2011.

27. Lu J, Yang Y, Chen L, Ren DF, Cai MY, Wang JZ, Egashira Y and Tanokura M: In vivo antihypertensive effect of Val-Glu-Pro in spontaneously hypertensive rats. Prog Biochem Biophys 38 : 353-360, 2011.

28. Majumder K, Chakrabarti S, Morton JS, Panahi S, Kaufman S, Davidge ST and Wu J: Egg-derived tri-peptide IRW exerts antihypert effects in spontaneously hypertensive rats. PLoS One 8 : e82829, 2013.

29. Bader $M$ and Ganten D: Update on tissue renin-angiotensin systems. J Mol Med (Berl) 86: 615-621, 2008.

30. Carey RM, Jin XH and Siragy HM: Role of the angiotensin AT2 receptor in blood pressure regulation and therapeutic implications. Am J Hypertens 14: 98S-102S, 2001.

31. Yu Z, Yin Y, Zhao W, Chen F and Liu J: Antihypertensive effect of angiotensin-converting enzyme inhibitory peptide RVPSL on spontaneously hypertensive rats by regulating gene expression of the renin-angiotensin system. J Agric Food Chem 62: 912-917, 2014.

32. Červenka L, Bíbová J, Husková Z, Vaňourková Z, Kramer HJ, Herget J, Jíchová Š, Sadowski J and Hampl V: Combined suppression of the intrarenal and circulating vasoconstrictor renin-ACE-ANG II axis and augmentation of the vasodilator ACE2-ANG 1-7-Mas axis attenuates the systemic hypertension in Ren-2 transgenic rats exposed to chronic hypoxia. Physiol Res 64: 11-24, 2015.
33. Mizuiri S and Ohashi Y: ACE and ACE2 in kidney disease. World J Nephrol 4: 74-82, 2015.

34. Soler MJ, Wysocki J and Batlle D: ACE2 alterations in kidney disease. Nephrol Dial Transpl 28: 2687-2697, 2013.

35. Kuba K, Imai Y and Penninger JM: Multiple functions of angiotensin-converting enzyme 2 and its relevance in cardiovascular diseases. Circ J 77: 301-308, 2013.

36. De Mello WC and Frohlich ED: On the local cardiac renin angiotensin system. Basic and clinical implications. Peptides 32: 1774-1779, 2011.

37. Patel VB, Clarke N, Wang Z, Fan D, Parajuli N, Basu R, Putko B, Kassiri Z, Turner AJ and Oudit GY: Angiotensin II induced proteolytic cleavage of myocardial ACE2 is mediated by TACE/ADAM-17: A positive feedback mechanism in the RAS. J Mol Cell Cardiol 66: 167-176, 2014

38. Patel KP and Schultz HD: Angiotensin peptides and nitric oxide in cardiovascular disease. Antioxid Redox Sign 19: 1121-1132, 2013.

39. Wolf $\mathrm{G}$ and Ritz E: Combination therapy with ACE inhibitors and angiotensin II receptor blockers to halt progression of chronic renal disease: Pathophysiology and indications. Kidney Int 67: 799-812, 2005. 